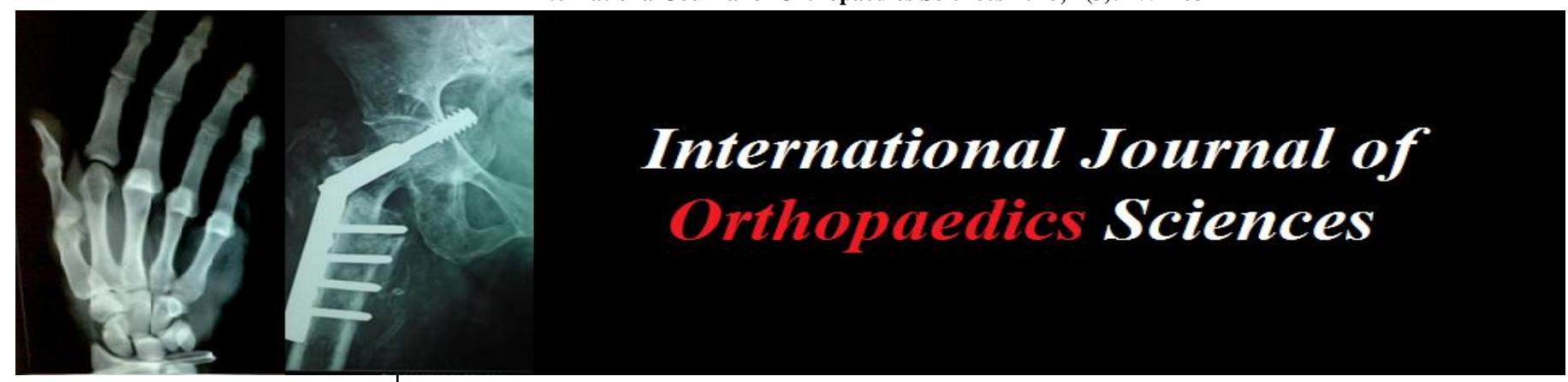

ISSN: $2395-1958$

IJOS 2018; 4(3): 259-263

(C) 2018 IJOS

www.orthopaper.com

Received: 15-05-2018

Accepted: 16-06-2018

Dr. Khaja Adil Ahmed

Ms(Ortho), Assistant Professor, Department of Orthopaedics,

Bhaskar Medical College,

Yenkapally, Moinabad, Ranga

Reddy, Telangana, India

Dr. Mir Zia Ur Rahman Ali Assistant Professor,

Department of Orthopaedics, Bhaskar Medical College,

Hyderabad, Telangana, India

Correspondence

Dr. Mir Zia Ur Rahman Ali

Assistant Professor,

Department of Orthopaedics,

Bhaskar Medical College,

Hyderabad, Telangana, India

\section{Comparative study of total knee replacement Vs non surgical treatment regarding management of knee osteoarthritis}

\author{
Dr. Khaja Adil Ahmed and Dr. Mir Zia Ur Rahman Ali
}

DOI: https://doi.org/10.22271/ortho.2018.v4.i3e.45

\section{Abstract}

Osteoarthritis (OA) is a type of joint disease that results from breakdown of joint cartilage and underlying bone. The most common symptoms are joint pain and stiffness. Osteoarthritis (OA) is a common disease of aged population and one of the leading causes of disability. Incidence of knee OA is rising by increasing average age of general population. Age, weight, trauma to joint due to repetiting movements in particular squatting and kneeling are common risk factors of knee OA. Several factors including cytokines, leptin, and mechanical forces are pathogenic factors of knee OA. Treatment options for osteoarthritis typically depend on the severity of the condition and the particular joints involved. They most often include medication Physical activity, stretching, and weight management, physical and occupational therapy, with or without assistive devices and surgery. The most common surgeries to treat knee arthritis are total knee replacement (total knee arthroplasty), partial knee replacement (unicompartmental knee arthroplasty), knee osteotomy (tibial osteotomy or femoral osteotomy), knee arthroscopy and cartilage repair and restoration. In this randomized, controlled trial, we enrolled 90 patients with moderate-to severe knee osteoarthritis who were eligible for unilateral total knee replacement. Patients were randomly assigned to undergo total knee replacement followed by 10 weeks of nonsurgical treatment (total-knee-replacement group) or to receive only the 10 weeks of nonsurgical treatment (nonsurgical-treatment group), which was delivered by physiotherapists and dietitians and consisted of exercise, education, dietary advice, use of insoles, and pain medication. The primary outcome was the change from baseline to 10 months in the mean score on four Knee Injury and Osteoarthritis Outcome Score subscales, covering pain, symptoms, activities of daily living, and quality of life (KOOS4); scores range from 0 (worst) to 100 (best). A total of 80 patients completed the 10month follow-up assessment. In the nonsurgical-treatment group, 11 patients underwent total knee replacement before the 10 -month follow-up; in the total-knee-replacement group, 1 patient received only nonsurgical treatment. In the intention-to-treat analysis, the total-kneereplacement group had greater improvement in the KOOS4 score than did the nonsurgical- treatment group. The total-knee-replacement group had a higher number of serious adverse events than did the nonsurgical-treatment group. In patients with knee osteoarthritis who were eligible for unilateral total knee replacement, treatment with total knee replacement followed by nonsurgical treatment resulted in greater pain relief and functional improvement after 10 months than did nonsurgical treatment alone. However, total knee replacement was associated with a higher number of serious adverse events than was nonsurgical treatment, and most patients who were assigned to receive nonsurgical treatment alone did not undergo total knee replacement before the 10-month follow-up.

Keywords: Knee, osteoarthritis, total knee replacement, nonsurgical treatment

\section{Introduction}

Osteoarthritis is the most common form of degenerative joint disease, and is a leading cause of disability in people over 50 years of age. It is can be a very debilitating form of arthritis in that it tends to affect the load-bearing joints, especially the hips and knees, that are crucial for normal movement. Osteoarthritis occurs when the cartilage cushioning the joints breaks down and causes the bones to rub together, inducing a change in shape. Osteoarthritis is found most frequently in people over age 50, although it can occur at any age. It most commonly affects joints in the knees, hips, shoulders, elbows, ankles, and hands. Gradual onset of knee pain, stiffness and swelling are typical symptoms of knee osteoarthritis. Arthritis of the knee comes 
in several forms. Osteoarthritis is by far the most common form, followed by some forms of inflammatory arthritis, such as rheumatoid arthritis and gout. There are several risk factors that make one more likely to develop the condition. The primary risk factors are advanced age (over age 45), prior knee injury, and excess weight. An arthritic knee has thinned, damaged or missing cartilage in the joint. The damaged cartilage is not in and of itself a source of pain or other symptoms. Instead, the damaged or missing cartilage causes friction between bones and other knee problems, which in turn cause knee pain and related symptoms. Knee pain and stiffness are classic signs of knee osteoarthritis. These symptoms tend to progress gradually and many people initially attribute knee soreness or discomfort to lack of exercise or getting older. Many dismiss their early knee arthritis symptoms as "no big deal" until they worsen and start to interrupt one's ability to enjoy everyday activities. Chief risk factors for developing knee osteoarthritis are advanced age and obesity. In addition to these, other risk factors for developing knee osteoarthritis include joint trauma, family history, congenital defect or illness, joint stress and chronic injury. While no single test can definitively diagnose osteoarthritis of the knee, physicians use a comprehensive approach that is verified by diagnostic imaging to arrive at a clinical diagnosis. It is important to clarify that most individuals over age 50 will have signs of osteoarthritis in their major joints that can be seen on an $\mathrm{x}$-ray, but most will have no symptoms, so the medical provider will not rely on diagnostic studies (such as an x-ray or MRI) alone. An accurate diagnosis will come following a comprehensive medical history and physical examination by the health care provider. While knee osteoarthritis is often a progressive and irreversible degenerative process, functional improvement and pain control are reasonable treatment goals. Earlier treatment intervention may improve the odds of preserving joint integrity and function for years - or even a lifetime - which can stave off debilitating pain and possible need for joint replacement surgery. Non-Surgical Treatments for Knee Osteoarthritis include physical therapy and exercise, Activity modification, Periodic rest, Warm or cold compress, Weight loss, Medications and injections for Knee Osteoarthritis (analgesics, non-steroidal anti-inflammatory drugs (NSAIDs), topical analgesics and Glucosamine and chondroitin sulfate; Two types of injections are commonly used for treatment of severe pain from knee osteoarthritis: steroid injections and hyaluronic acid injections), Supportive devices and orthotics, Coping techniques. Generally, any knee surgery to alleviate arthritis pain is considered elective surgery, meaning it is up to the patient to decide whether or not to have surgery. Typically, patients who have knee arthritis consider surgery when quality of life is significantly affected because of pain and functional limitations, non-surgical solutions to control pain, such as medication and physical therapy, have been tried for several months or longer without satisfactory improvement, an X-ray or MRI confirms knee joint degeneration. The most common surgeries to treat knee arthritis are total knee replacement (total knee arthroplasty), partial knee replacement (unicompartmental knee arthroplasty), knee osteotomy (tibial osteotomy or femoral osteotomy), knee arthroscopy and cartilage repair and restoration. Total Knee Replacement Surgery (Total Knee Arthroplasty): Patients with moderate to severe knee arthritis who have exhausted non-surgical treatment options might consider replacing the entire knee joint. The surgery involves cutting the arthritically damaged ends of the tibia (shin bone) and femur (thighbone) and capping both with prostheses (like capping teeth). The two prosthetic pieces are made of either metal or a durable plastic called polyethylene. These new surfaces move smoothly against one another, creating a functional knee joint. Partial recovery typically takes 4 to 6 weeks 1 and full recovery may take a few months to an entire year. Total knee replacement surgery provides most patients with pain relief and improved knee joint function. However, a replacement knee is not as strong and durable as a healthy knee, and patients are strongly advised not to engage in highimpact activities (such as jogging) that may speed up the wear-and-tear of the new joint. In this randomized, controlled trial, we enrolled 90 patients with moderate-to severe knee osteoarthritis who were eligible for unilateral total knee replacement. Patients were randomly assigned to undergo total knee replacement followed by 10 weeks of nonsurgical treatment (total-knee-replacement group) or to receive only the 10 weeks of nonsurgical treatment (nonsurgical-treatment group), which was delivered by physiotherapists and dietitians and consisted of exercise, education, dietary advice, use of insoles, and pain medication. The primary outcome was the change from baseline to 10 months in the mean score on four Knee Injury and Osteoarthritis Outcome Score subscales, covering pain, symptoms, activities of daily living, and quality of life (KOOS4); scores range from 0 (worst) to 100 (best). A total of 80 patients completed the 10-month followup assessment.

\section{Patients and Methods}

Study design : This is a prospective cross-sectional study.

Approval : This study received approval from college ethical committee.

Setting : Hyderabd.
Participants : Ninety patients admitted in Hospital.
Study period : June 2016 - December 2017

\section{Observations and Results}

Enrollment and Follow-up: A total of 90 patients underwent randomization 43 of 45 patients in the nonsurgical- treatment group and 40 of 45 patients in the total-knee-replac ement group completed the 10-month follow-up assessment. In the nonsurgical- treatment group, 11 of 45 patients had a total knee replacement before the 10-month follow-up. In the totalknee-replacement group, 1 of 45 patients decided not to undergo total knee replacement and received only the nonsurgical treatment. All 90 patients were included in the intention-to-treat analysis, whereas 23 of 44 patients in the nonsurgical-treatment group and 26 of 41 patients in the totalknee- replacement group were included in the perprotocol analysis. 
Table 1: Baseline Characteristics of the Patients.

\begin{tabular}{|c|c|c|}
\hline & \multirow{2}{*}{$\frac{\text { Nonsurgical- }}{\text { Treatment }}$} & Total-Knee- \\
\hline & & Replacement \\
\hline & Group & Group \\
\hline Characteristic & $(N=45)$ & $(N=45)$ \\
\hline Female sex - no. $(\%)$ & \begin{tabular}{l|l}
27 &
\end{tabular} & 28 \\
\hline Age $-\mathrm{yr}$ & $63.0 \pm 8.7$ & $64.7 \pm 8.7$ \\
\hline Body-mass index $\dagger$ & $31.0 \pm 4.5$ & $31.4 \pm 6.2$ \\
\hline \multicolumn{3}{|l|}{ Kellgren-Lawrence score - no. $(\%)$} \\
\hline 2 & 5 & 7 \\
\hline 3 & 20 & 21 \\
\hline 4 & 23 & 22 \\
\hline \multicolumn{3}{|l|}{ KOOS scores§ } \\
\hline $\mathrm{KOOS}_{4}$ & $46.5 \pm 11.4$ & $47.4 \pm 13.4$ \\
\hline Pain & $49.5 \pm 13.1$ & $48.6 \pm 17.5$ \\
\hline Symptoms & $58.6 \pm 15.2$ & $54.0 \pm 15.0$ \\
\hline Activities of daily living & $52.5 \pm 14.2$ & $55.0 \pm 17.0$ \\
\hline Quality of life & $32.7 \pm 13.3$ & $32.3 \pm 15.3$ \\
\hline Sports and recreation & $17.7 \pm 15.1$ & $18.0 \pm 14.7$ \\
\hline Time on the timed up-and-go test $-\mathrm{sec}$ & $8.6 \pm 2.1$ & $9.4 \pm 2.4$ \\
\hline Time on the $20-\mathrm{m}$ walk tests $-\mathrm{sec}$ & $12.1 \pm 2.5$ & $13.4 \pm 3.7$ \\
\hline \multicolumn{3}{|l|}{ EQ-5D scoresय } \\
\hline Descriptive index & $0.661 \pm 0.147$ & $0.661 \pm 0.156$ \\
\hline Visual-analogue scale & $63.8 \pm 16.1$ & $66.3 \pm 17.2$ \\
\hline Used pain medication in the past week & \begin{tabular}{l|l}
24 & \\
\end{tabular} & 28 \\
\hline
\end{tabular}

In the intention-to-treat analysis, the total-knee replacement group had a significantly greater improvement in the KOOS4 score than did the nonsurgical-treatment group, with a crude mean difference of 16.5. In the nonsurgicaltreatment group, the increase in the KOOS4 from baseline to month 10 was 16.0. Furthermore, as compared with the nonsurgical- treatment group, the total-knee-replacement group had significantly greater improvements in the scores on all five KOOS subscales, the times on the timed up-andgo test and 20-m walk tests, and the scores on the EQ-5D descriptive index.

Table 2: Outcomes at 12 Months

\begin{tabular}{|c|c|c|c|c|c|c|}
\hline & & & \multirow{2}{*}{\multicolumn{2}{|c|}{$\begin{array}{l}\text { Mean Improvement in Outcome } \\
\text { from Baseline to } 10 \mathrm{Mo}\end{array}$}} & \multirow{2}{*}{\multicolumn{2}{|c|}{$\begin{array}{c}\text { Between-Group Difference in } \\
\text { Mean Improvement }\end{array}$}} \\
\hline & & & & & & \\
\hline \multirow[t]{4}{*}{ Outcome } & \multicolumn{2}{|c|}{ Total No. of Assessments* } & \multicolumn{2}{|c|}{$(95 \% \mathrm{CI})$} & \multicolumn{2}{|c|}{$(95 \% \mathrm{CI})$} \\
\hline & \multicolumn{2}{|c|}{ Total-Knee- Nonsurgical- } & Nonsurgical- & Total-Knee- & & \\
\hline & Replacement & Treatment & Treatment & Replacement & & \\
\hline & Group & Group & Group & Group & Crude & Adjusted $\dagger$ \\
\hline \multicolumn{7}{|l|}{ Primary outcome } \\
\hline \multirow[t]{2}{*}{$\mathrm{KOOS}_{4}$} & 168 & 189 & 16.1 & 31.5 & 16.5 & 14.4 \\
\hline & & & (10.1 to 21.9$)$ & (26.6 to 38.3$)$ & (10.2 to 22.7$)$ & $(10.0$ to 21.5$)$ \\
\hline \multicolumn{7}{|l|}{ Secondary outcomes } \\
\hline \multicolumn{7}{|l|}{ KOOS subscale scores } \\
\hline \multirow[t]{2}{*}{ Pain } & 178 & 190 & 17.2 & 32.7 & 17.6 & 15.3 \\
\hline & & & (10.4 to 24.1$)$ & (28.1 to 41.5$)$ & (10.1 to 25.1$)$ & (10.4 to 23.8$)$ \\
\hline \multirow[t]{2}{*}{ Symptoms } & 172 & 194 & 11.4 & 26.4 & 15.0 & 12.7 \\
\hline & & & (4.4 to 18.4$)$ & (21.5 to 31.4$)$ & (8.3 to 21.7$)$ & (6.6 to 17.6$)$ \\
\hline \multirow[t]{2}{*}{ Activities of daily living } & 170 & 193 & 17.6 & 30.1 & 12.3 & 12.9 \\
\hline & & & (11.4 to 23.9$)$ & (22.7 to 37.2$)$ & (5.5 to 19.2$)$ & (6.8 to 19.1$)$ \\
\hline \multirow[t]{2}{*}{ Quality of life } & 175 & 194 & 17.8 & 38.2 & 20.4 & 20.2 \\
\hline & & & (11.2 to 24.4$)$ & (30.6 to 45.8$)$ & $(12.8$ to 27.9$)$ & $(13.2$ to 27.1$)$ \\
\hline \multirow[t]{2}{*}{ Sports and recreation } & 171 & 193 & 19.3 & 32.3 & 15.2 & 15.6 \\
\hline & & & (10.8 to 27.7$)$ & (27.9 to 40.0$)$ & (6.7 to 23.7$)$ & (7.3 to 22.2 ) \\
\hline Time on the timed up-and-go test & 156 & 185 & -1.1 & -2.2 & 1.2 & 0.8 \\
\hline$(\mathrm{sec})$ & & & $(-1.8$ to -0.6$)$ & $(-3.1$ to -1.6$)$ & (0.4 to 1.9$)$ & $(0.2$ to 1.5$)$ \\
\hline \multirow[t]{2}{*}{ Time on the $20-\mathrm{m}$ walk tests $(\mathrm{sec})$} & 160 & 185 & -1.0 & -2.5 & 1.9 & 1.5 \\
\hline & & & $(-1.5$ to -0.4$)$ & $(-3.8$ to -1.9$)$ & $(0.9$ to 2.8$)$ & $(0.7$ to 2.4$)$ \\
\hline \multicolumn{7}{|l|}{ EQ-5D scores } \\
\hline \multirow[t]{2}{*}{ Descriptive index } & 178 & 189 & 0.118 & 0.206 & 0.091 & 0.078 \\
\hline & & & (0.061 to 0.156$)$ & (0.141 to 0.270$)$ & (0.026 to 0.155$)$ & $(0.023$ to 0.132$)$ \\
\hline \multirow[t]{2}{*}{ Visual-analogue scale } & 180 & 193 & 10.2 & 15.0 & 4.9 & 4.4 \\
\hline & & & (4.6 to 15.7 ) & (8.6 to 21.5$)$ & $(-2.2$ to 12.0$)$ & $(-1.8$ to 10.6$)$ \\
\hline \multirow[t]{2}{*}{ Weight $(\mathrm{kg}) \dagger$} & 134 & 160 & -2.6 & 0.1 & 2.8 & 2.8 \\
\hline & & & $(-3.9$ to -1.4$)$ & $(-1.5$ to 1.7$)$ & (1.4 to 4.1$)$ & (1.4 to 4.1$)$ \\
\hline
\end{tabular}




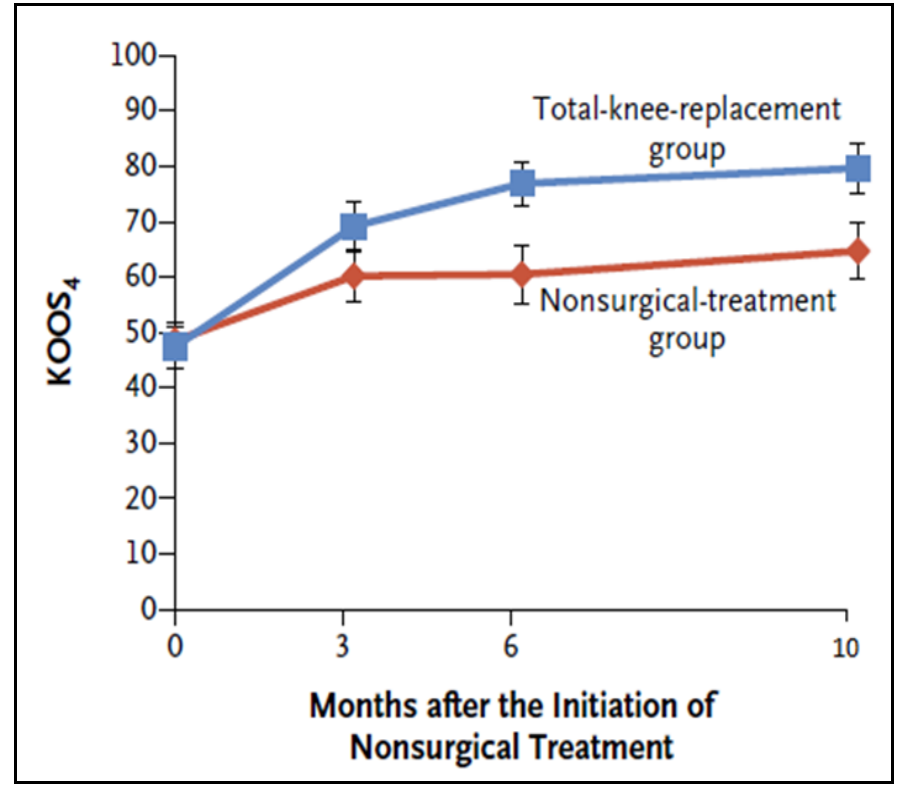

Fig 1: Primary Outcome.
Serious adverse events were more common in the total-kneereplacement group than in the nonsurgical-treatment group. In the total-knee-replacement group, the two most common serious adverse events involving the index knee were deep venous thrombosis (in 2 patients) and stiffness requiring brisement forcé (in 2 patients). The per-protocol analysis also showed that the total-knee-replacement group had a significantly higher increase in the KOOS4 than did the nonsurgical-treatment group. The per-protocol analysis of the secondary outcomes yielded results similar to those of the intention-to-treat analysis, except that there was a significant between group difference in the scores on the EQ-5D visualanalogue scale and not in the scores on the KOOS symptoms subscale. In the as-treated analysis of adverse events, serious adverse events were more likely to occur after total knee replacement had been performed than before. The number needed to treat with total knee replacement for a $15 \%$ improvement from baseline to 10 months in KOOS4 was 5.7 in the intention-to-treat analysis and 6.0 in the per-protocol analysis.

Table 2: Serious Adverse Events.

\begin{tabular}{|c|c|c|c|}
\hline & \multicolumn{2}{|c|}{ Nonsurgical- Total-Knee- } & \multirow{3}{*}{\begin{tabular}{|c|}
$\mathbf{P}$ \\
Value \\
\end{tabular}} \\
\hline & Treatment & Replacement & \\
\hline \multirow[t]{2}{*}{ Events } & Group & Group & \\
\hline & \multicolumn{2}{|c|}{ no. of events } & \\
\hline Overall & 6 & 24 & 0.005 \\
\hline Involving sites other than the index knee & 5 & 16 & 0.04 \\
\hline Musculoskeletal & 0 & 4 & \\
\hline Skin & 1 & 0 & \\
\hline Gastrointestinal & 0 & 3 & \\
\hline Other & 4 & 9 & \\
\hline Involving the index knee & 1 & 8 & 0.05 \\
\hline Occurred during total knee replacement & 0 & 0 & \\
\hline \multicolumn{4}{|l|}{ Occurred after total knee replacement } \\
\hline Stiffness requiring brisement forcé $\dagger$ & 1 & 1 & \\
\hline Deep infection & 0 & 1 & \\
\hline Deep venous thrombosis requiring & 0 & 1 & \\
\hline \multicolumn{4}{|l|}{ anticoagulation } \\
\hline Supracondylar femur fracture & 0 & 1 & \\
\hline
\end{tabular}

\section{Discussion}

This randomized, controlled trial showed that total knee replacement followed by nonsurgical treatment is more efficacious than nonsurgical treatment alone in providing pain relief and improving function and quality of life after 10 months in patients with knee osteoarthritis who are eligible for unilateral total knee replacement. However, clinically relevant improvements were noted in both groups, and patients who underwent total knee replacement had a higher number of serious adverse events. We are not aware of any previous or ongoing randomized trials investigating the effectiveness of total knee replacement, despite its wide and increasing use. Previous reports on the effects of total knee replacement have been case series, without a control group for comparison. Both groups in our study had substantial improvement with respect to most outcomes, and only $22 \%$ of the patients who were assigned to receive nonsurgical treatment alone underwent total knee replacement in the following year. Previous reports have suggested a benefit of nonsurgical treatment in patients with moderateto- severe knee osteoarthritis who are eligible for total knee replacement. Even for patients progressing to surgery, participation in supervised exercise before surgery has been associated with a faster postoperative recovery. The benefits and harms of the respective treatments underscore the importance of considering patients' preferences and values during shared decision making about treatment for moderateto-severe knee osteoarthritis. Our study has limitations. We did not include a sham-surgery control group; since surgery and, to a lesser extent, nonsurgical treatments are associated with placebo effects, the findings in this study may overestimate effects attributable to the specific treatments and to surgery in particular. The scores on the KOOS pain subscale that were obtained before surgery were similar to those obtained in previous studies of total knee replacement 39,40 and indicated mildto- severe pain during activities, but it is not known whether our results are generalizable to patients with more severe pain. The intensity of nonsurgical treatment may have differed between groups owing to differences in clinical status at the time treatment was initiated. However, the intervention was standardized and administered in both groups by the same physiotherapists and dietitians. Since all patients received multimodal nonsurgical treatment, it is not possible to separate the effects of the individual modes of treatment. The combination of nonsurgical treatments that we administered complies with international recommendations on the treatment of knee osteoarthritis, which increases the generalizability of the 
results.

\section{Conclusions}

In conclusion, our results show that total knee replacement followed by nonsurgical treatment is superior to nonsurgical treatment alone in providing pain relief and improving function and quality of life after 10 months in patients with moderate-to-severe knee osteoarthritis who are eligible for unilateral total knee replacement. However, total knee replacement is associated with a higher number of serious adverse events, and most patients who were assigned to receive nonsurgical treatment alone did not undergo total knee replacement before the 10-month follow-up and had clinically relevant improvements.

\section{References}

1. Kurtz S, Ong K, Lau E, Mowat F, Halpern M. Projections of primary and revision hip and knee arthroplasty in the The New England Journal of Medicine

2. Lim HC, Adie S, Naylor JM, Harris IA. Randomised trial support for orthopaedic surgical procedures. PLoS One. 2014; 9(6):e96745.

3. Fernandes L, Hagen KB, Bijlsma JW. EULAR recommendations for the non-pharmacological core management of hip and knee osteoarthritis. Ann Rheum Dis. 2013; 72:1125-35.

4. McAlindon TE, Bannuru RR, Sullivan MC. OARSI guidelines for the nonsurgical management of knee osteoarthritis. Osteoarthritis Cartilage. 2014; 22:363-88.

5. Nelson AE, Allen KD, Golightly YM, Goode AP, Jordan JM. A systematic review of recommendations and guidelines for the management of osteoarthritis: the Chronic Osteoarthritis Management Initiative of the U.S. Bone and Joint Initiative. Semin Arthritis Rheum. 2014; 43:701-12.

6. Skou ST, Roos EM, Laursen MB. Total knee replacement plus physical and medical therapy or treatment with physical and medical therapy alone: A randomized controlled trial in patients with knee osteoarthritis (the MEDIC-study). BMC Musculoskelet Disord. 2012; 13:67.

7. Moher D, Hopewell S, Schulz KF. CONSORT 2010 explanation and elaboration: updated guidelines for reporting parallel group randomised trials. BMJ. 2010; 340: c869.

8. Schiphof D, de Klerk BM, Kerkhof HJ. Impact of different descriptions of the Kellgren and Lawrence classification criteria on the diagnosis of knee osteoarthritis. Ann Rheum Dis. 2011; 70:1422-7.

9. Endres S. High-flexion versus conventional total knee arthroplasty: a 5-year study. J Orthop Surg (Hong Kong). 2011; 19:226-9.

10. Skou ST, Rasmussen S, Laursen MB. The efficacy of 12 weeks non-surgical treatment for patients not eligible for total knee replacement: a randomized controlled trial with 1-year follow-up. Osteoarthritis Cartilage. 2015; 23:1465-75.

11. Hayami T. Osteoarthritis of the knee joint as a cause of musculoskeletal ambulation disability symptom complex (MADS) Clin Calcium. 2008; 18:1574-80.

12. Peat G, McCarney R, Croft P. Knee pain and osteoarthritis in older adults: a review of community burden and current use of primary health care. Ann Rheum Dis. 2001; 60:91-7.

13. Zhang Y, Jordan JM. Epidemiology of osteoarthritis. Clin
Geriatr Med. 2010; 26:355-69.

14. Srikanth VK, Fryer JL, Zhai G. A meta-analysis of sex differences prevalence, incidence and severity of osteoarthritis. Osteoarthritis Cartilage. 2005; 13:769-81.

15. Sowers M, Karvonen-Gutierrez CA, Jacobson JA, Jiang Y, Yosef M. Associations of anatomical measures from MRI with radiographically defined knee osteoarthritis score, pain, and physical functioning. J Bone Joint Surg Am. 2011; 93:241-51.

16. Muraki S, Oka H, Akune T. Prevalence of radiographic knee osteoarthritis and its association with knee pain in the elderly of Japanese population-based cohorts: the ROAD study. Osteoarthritis Cartilage. 2009; 17:1137-43.

17. Andrianakos AA, Kontelis LK, Karamitsos DG. Prevalence of symptomatic knee, hand, and hip osteoarthritis in Greece. The ESORDIG study. J Rheumatol. 2006; 33:2507-13.

18. Jordan JM, Helmick CG, Renner JB. Prevalence of knee symptoms and radiographic and symptomatic knee osteoarthritis in African Americans and Caucasians: the Johnston County Osteoarthritis Project. J Rheumatol. 2007; 34:172-80.

19. Bedson J, Croft PR. The discordance between clinical and radiographic knee osteoarthritis: a systematic search and summary of the literature. BMC Musculoskelet Disord. 2008; 9:116.

20. Peat G, Thomas E, Duncan R. Estimating the probability of radiographic osteoarthritis in the older patient with knee pain. Arthritis Rheum. 2007; 15:794-802.

21. Cicuttini FM, Baker J, Hart DJ, Spector TD. Association of pain with radiological changes in different compartments and views of the knee joint. Osteoarthritis Cartilage. 1996; 4:143-7.

22. Hayes CW, Jamadar DA, Welch GW. Osteoarthritis of the knee: comparison of MR imaging findings with radiographic severity measurements and pain in middleaged women. Radiology. 2005; 237:998-1007.

23. Fukui N, Yamane S, Ishida S. Relationship between radiographic changes and symptoms or physical examination findings in subjects with symptomatic medial knee osteoarthritis: a three-year prospective study. BMC Musculoskelet Disord. 2010; 11:26917.

24. Thorp LE, Sumner DR, Wimmer MA, Block JA. Relationship between pain and medial knee joint loading in mild radiographic knee osteoarthritis. Arthritis Rheum. 2007; 57:1254-60. 